Bettina Habsburg-Lothringen

\title{
Ein Waffenlager, ein Fotoarchiv, ein Schaudepot
}

\section{Museen und die Masse der Dinge}

Schauplatz Archiv. Objekt, Narrativ, Performanz. Von der Begriffsfolge, die den Gegenstand dieses Tagungsbandes umschreibt, ist der des Archivs in musealen Kontexten am wenigsten gebräuchlich: Von Archiv ist bestenfalls die Rede, wenn es um Dokumente und Belege zur Institutionengeschichte geht. Im „Sacharchiv“ (Groys 2005, 39) deutet sich zudem eine der zentralen gesellschaftlichen Funktionen der Institution an, die Überreste und Hinterlassenschaften vergangener Zeiten zu sammeln, zu erhalten und für Vergleiche zugänglich zu machen. Das Archiv des Museums im Sinne von „Lager“ und „nichtöffentlicher Schutzraum“ ist das Depot. Dieses hat in den musealen Debatten der letzten Jahre an Präsenz gewonnen. Als Zentrum hinter den Kulissen, als Herz der Institution war es Gegenstand alltagspraktischer Debatten rund um Ausstattung, Klima oder sicherheitstechnische Standards. Nun wird das, was lang verborgen war, als potenziell zugänglicher Ort und Konzept diskutiert.

Wie steht es um das Verhältnis des historischen Museums zum Depot? Depots gibt es in den Museen nicht von Beginn an in der uns heute bekannten Form. Ihre Existenz verdankt sich der Entwicklung der Wissenschaften und der wandelnden institutionellen Identität im Verlauf des 19. Jahrhunderts. Die Kunst- und Wunderkammern waren Schauraum und Lager, Sammlungs- und Präsentationsort in einem. Hier fand sich dicht gedrängt, was die Diversität der bekannten Wirklichkeit bestimmte und dem persönlichen Geschmack des Sammlers entsprach. Zur Sicherung einzelner Objekte dienten Schränke und Truhen, Regale und Kredenzen innerhalb der Kammern. Diese fanden sich auch in den frühen Museen, wo Räume und Säle mit dem Ziel angelegt wurden, die gesamte Natur und Kultur darin zu versammeln und die Welt gänzlich zu dokumentieren. Der repräsentative architektonische Rahmen für diese Gesamtschauen war als ein endgültiger und nicht auf Wechsel oder Expansion angelegt.

Im 19. Jahrhundert ließ der rasante Zuwachs an Wissen die Idee einer Aneignung der Welt in enzyklopädischer Form zur musealen Illusion verkommen. Immer neue Forschungserkenntnisse führten zur Ausdifferenzierung der Wissenschaften und in den Museen zu einer enormen Anhäufung von Belegen. Es entstanden Spezialgebiete mit neuen Fragestellung und - so Hanno Möbius - mit ihnen der Zweifel an der Möglichkeit, die Welt in Sälen, seien diese noch so groß, 
je fassen zu können (vgl. Möbius 2006, 14). Eine Folge der stetig wachsenden Sammlungen war die Abtrennung der Sammlungsdepots von den Ausstellungsräumen, mit Folgen für die Architektur und Raumausstattung: Anke te Heesen beschreibt, dass der klassische Sammlungs- und Präsentationsschrank des 18. Jahrhunderts im oberen Teil mit Glas versehen und im unteren Drittel mit einem durch Türen geschützten Stauraum ausgestattet war (vgl. Heesen 2001, 31). Im 19. Jahrhundert wurde daraus die Vitrine, die ausgewählte Stücke der Natur, der Geschichte, der Völkerkunde und des Kunstgewerbes in wissenschaftlich motivierter Ordnung und gut überblickbar für das Publikum präsentierte. Für einen Gutteil der Objekte entstanden Depots und Labore, deren Gestaltung - weil für das Publikum nicht zugänglich und so keinen didaktischen Forderungen unterworfen - neuen Ordnungen und Regeln folgten.

Mit dem Ausbau und der Diversifizierung des Museumswesens wurde der private Sammler zum Wissenschaftler im Museumshintergrund und der Staat zum Träger einer in mehrerlei Hinsicht „nützlichen“ Institution. Die Erforschung von Natur, Kunst und Geschichte sowie der fachliche Austausch darüber spielten sich von nun an hinter den Kulissen ab. Ziel der wissenschaftlichen Museumsarbeit war es, den Erkenntnisstand der Disziplinen voranzubringen und eine Grundlage für die Vermittlungsarbeit des Museums in Form von Ausstellungen oder Bildungsprogrammen zu schaffen. Die Staaten und weitere öffentliche Trägerinstitutionen wollten mehr: mit Geschichte, Kunst, Natur und Technik Identitäts- und Orientierungsangebote bereitstellen, instrumentelles Wissen zur positiven Entwicklung von Landwirtschaft, Handwerk und Industrie sowie Sachwissen zur Alltagsbewältigung anbieten. Sich selbst feiern: Wie Sharon McDonald ausführt, bezeugten die umfangreichen, wohlgeordneten Darstellungen, die properen Vitrinen- und Schubladenpräsentationen im prunkvollen architektonischen Rahmen eine kulturelle, moralische und technologische Überlegenheit der Museumsträger und die Fähigkeit der Museumsverantwortlichen, Kontrolle - gleich ob über Natur, Geschichte oder andere Völker - auszuüben (vgl. McDonald 2003, 3).

In welchem Verhältnis stehen nun Depot und Ausstellungsraum nach diesem Zerfallen des Museums in eines vor und eines hinter den Kulissen? Das Depot ist von nun an Speicher und Vorratsraum. Es steht für das Ganze, die über Generationen gewachsene Fülle, aus der je nach Zeit, wissenschaftlichen Interessen und persönlichen Neigungen der Verantwortlichen einzelne Objekte herausgenommen und zur Veranschaulichung historischer Sachverhalte, kultureller Prozesse oder kunstgeschichtlicher Entwicklungen eingesetzt werden. In welchen Kontexten der Begriff Depot in der aktuellen Museumspraxis darüber hinaus noch Verwendung findet, zeigt exemplarisch der Blick auf ein Stück Grazer Museumsalltag und die Abteilung für Kulturgeschichte am Universalmuseum Joanneum, die neben dem Landeszeughaus und dem Volkskundemuseum auch das Museum für 
Geschichte mit der Kulturhistorischen Sammlung und den Multimedialen Sammlungen umfasst. Depots prägen den Arbeitsalltag der Abteilung in mehrfacher und sehr unterschiedlicher Weise.

Einmal im Landeszeughaus. Die Anfänge des Landeszeughauses gehen auf das 17. Jahrhundert zurück: Die Zeit vom 15. bis zum 18. Jahrhundert war für die damals innerösterreichischen Länder durch kriegerische Auseinandersetzungen mit ungarischen Rebellen und dem Osmanischen Reich geprägt. Vor diesem Hintergrund veranlassten die steirischen Landstände Mitte des 17. Jahrhunderts den Bau des Zeughauses, das als Waffendepot die wichtigste „Ausrüstungs-Zentrale“ im Südosten des habsburgischen Reichs darstellte. Mit dem Rückgang der bewaffneten Konflikte im 18. Jahrhundert verlor das Zeughaus seine Funktion, und als Maria Theresia im Zuge einer Reform beschloss, das Zeughaus aufzulösen, erbaten die Landstände seine Erhaltung als Denkmal der Landesgeschichte. 1882 wurde das landschaftliche Zeughaus erstmals der Öffentlichkeit zugänglich gemacht, 1892 in das von Erzherzog Johann gestiftete Joanneum eingegliedert.

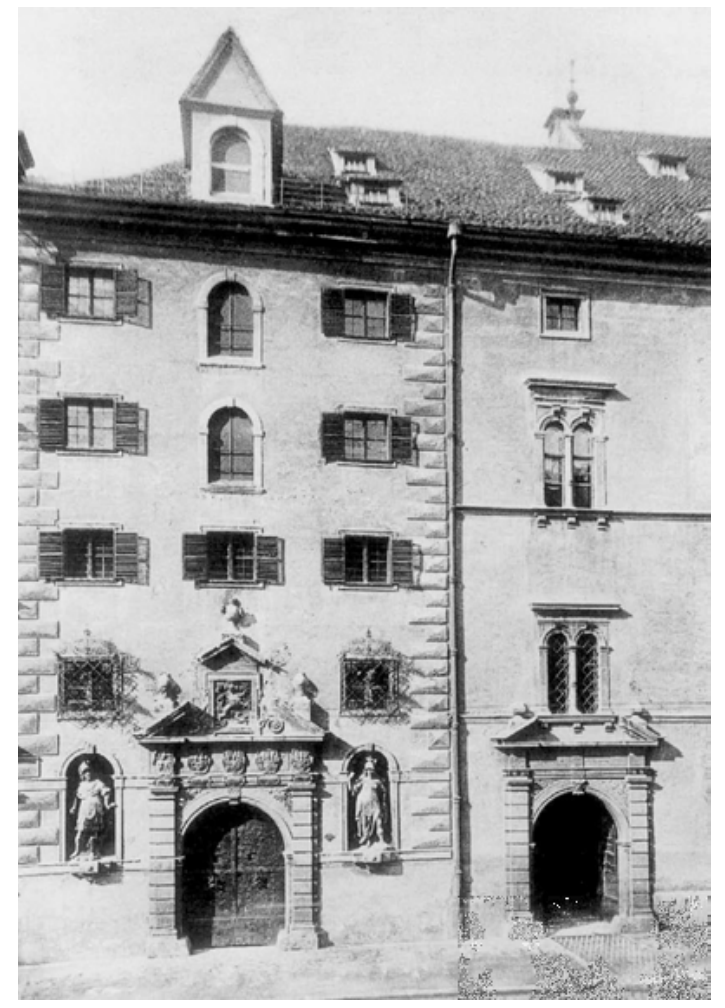

Abb. 1: Älteste Aufnahme des landschaftlichen Zeughauses, 1845. 
Die heute erhaltene Sammlung des Hauses umfasst gut 32.000 Harnische und Waffen, Kanonen und Pulverhörner, präsentiert auf vier Etagen und rund 2.000 Quadratmetern Fläche. Der Objektbestand stellt dabei lediglich einen Rest dar: Um 1700 erreichte die Zahl der beherbergten Kriegsgeräte mit beinahe 190.000 Stück ihren Höhepunkt.

Ein Depot anderer Form ist das Schaudepot der Kulturhistorischen Sammlung im Museum für Geschichte. Im späten 19. Jahrhundert mit dem Ziel begründet, die Kulturepochen des Landes und sein kunstgewerbliches Erbe zu dokumentieren, ist die Sammlung eine typische Zeitgeist-Erscheinung: Industrialisierung und Massenproduktion beförderten im späten 19. Jahrhundert überall in Europa das Bedürfnis, die Spuren einer verschwindenden Welt museal zu sichern, und führten zur Anlage entsprechender Sammlungen und Museen, die sich im Verlauf des 20. Jahrhunderts im Regelfall entweder zu kulturhistorischen Spezialmuseen oder zu Designmuseen weiterentwickelten. Bedingt durch Umbauten und schließlich den Umzug der Kulturhistorischen Sammlung ins Palais Herberstein, war es in Graz ab den 1990er-Jahren nicht mehr möglich, einen umfassenderen Einblick in die rund 35.000 Objekte umfassende Sammlung zu erhalten. Seit 2017 ermöglicht dies ein Schaudepot, das die Bestände auf einer Fläche von rund 500 Quadratmetern repräsentativ als dichte Collage von rund 2.000 Objekten vorstellt.

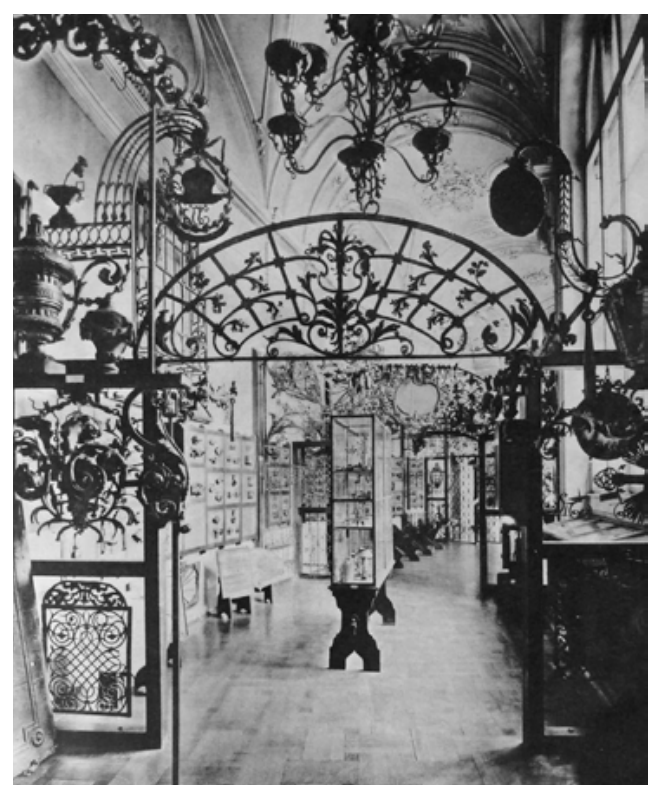

Abb. 2: Culturhistorisches und Kunstgewerbemuseum, Eingang zur Schmiedeeisensammlung, 1895. 
Dem klassischen Depot am nächsten kommt jenes der Multimedialen Sammlungen, die mit rund 2,5 Millionen Fotografien, Film- und Tondokumenten von gleichermaßen medienhistorischer, zeit- und regionalgeschichtlicher Relevanz sind. Die Anfänge der Sammlung, die als Bild- und Tonarchiv, Servicestelle und Ort der Produktion von Medienobjekten konzipiert wurde, gehen auf die späten 1950er-Jahre zurück. Bis zum Ende der 1980er-Jahre war die Sammlung bereits auf Hunderttausende Negative, Positivkopien auf Papier, Diapositive, Tonbänder, Kassetten, Filmkopien und Videokassetten angewachsen. Der Versuch, die große Zahl der so unterschiedlichen Objekte konservatorisch adäquat unterzubringen, führte zu gleich mehreren Umzügen innerhalb einer vergleichsweise kurzen Sammlungsgeschichte. Mit der schrittweisen Anerkennung von Fotografie, Film und Ton als vollwertige museale Objekte wurde aus dem Medienarchiv eine museale Sammlung, die heute im Museum für Geschichte auszugsweise präsentiert wird, während ein Gutteil der Bestände in Klimadepots lagert.

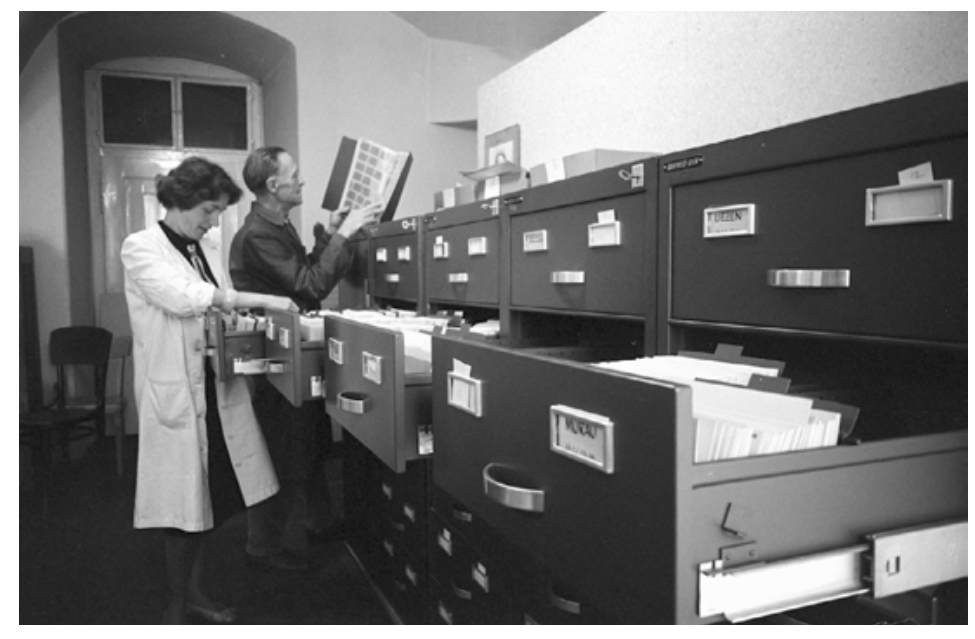

Abb. 3: Film- und Tonarchiv.

Wodurch unterscheiden sich nun diese Depots, die sich in ihrer äußeren Erscheinung durchaus ähneln, voneinander? Das Landeszeughaus ist Relikt einer konfliktreichen Zeit und Teil des Landesgedächtnisses. Als historischer Ort ist es nicht Tat- oder Leidensort, nicht Gedenkstätte oder Mahnmal, wie viele der im 20. Jahrhundert im Rahmen des Opfergedenkens musealisierten Stätten und Einrichtungen der Gewalt und des Krieges. Es ist ein historisch gewachsenes Waffenlager mit geschlossener Sammlung, deren Aufstellung dem Schutz des Denkmalamtes unterliegt. Es kommt ohne Objektbeschriftungen und Vitrinen aus, ein 
Umstand, der vom Publikum ein hohes Maß an Disziplin erfordert, aber den musealen Charakter der Einrichtung nicht zu mindern scheint: Der Gutteil der BesucherInnen nimmt das Landeszeughaus als Museum wahr, obwohl es das in entscheidenden Punkten nicht ist. Im Museum der Moderne finden sich absichtsvoll zusammengetragene Objekte, die bewahrt und beforscht werden, um mit ihrer Hilfe historische Ereignisse und Entwicklungen einer breiten Öffentlichkeit vorzustellen. Museale Sammlungsbestände zeichnen sich dadurch aus, dass sie, aus ihrem ursprünglichen Gebrauchskontext und Verwendungszusammenhang herausgenommen, im Museum stillgestellt werden und als Träger von Information und Bedeutung für etwas stehen, das außerhalb ihrer selbst liegt. So lassen sich beispielsweise an historischen Möbeln kunsthandwerkliche Techniken und die Moden einer Zeit ablesen, Wirtschafts- und Handelsbeziehungen nachvollziehen oder Erkenntnisse über die Praktiken der Nutzung und die Sozialgeschichte der Produktion gewinnen.

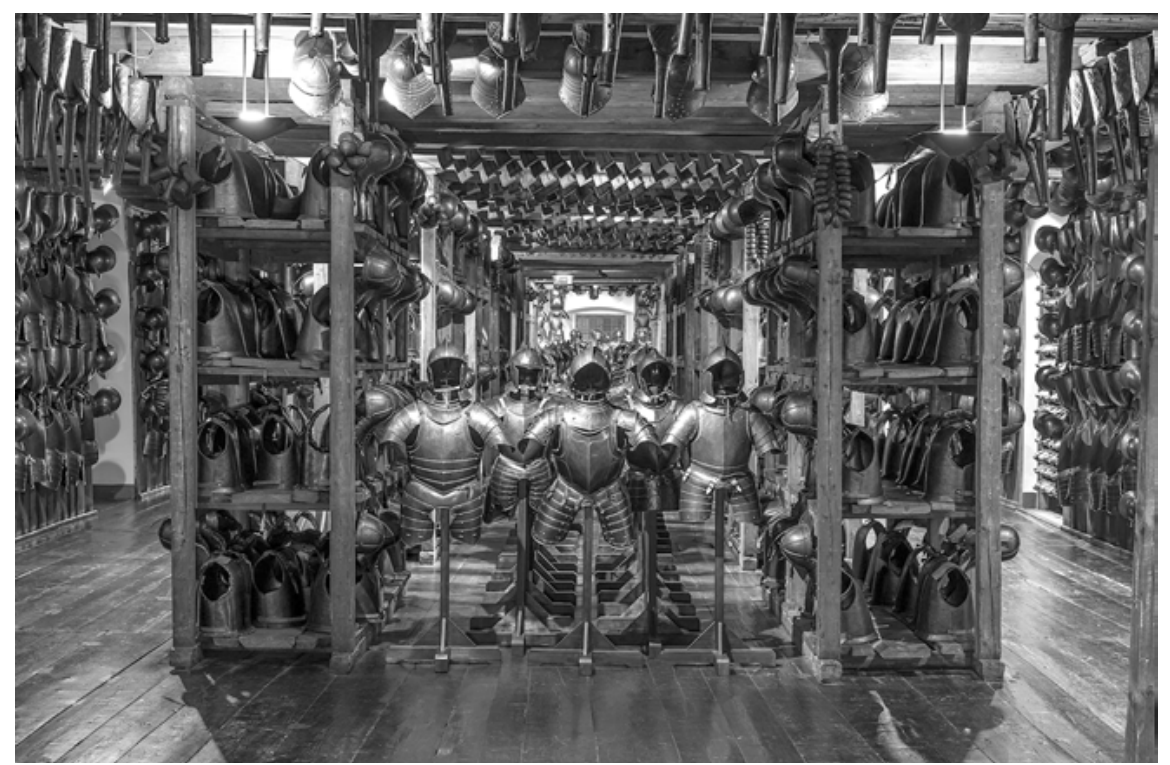

Abb. 4: Landeszeughaus.

Das Zeughaus ist durchaus ein Ort der absichtsvoll zusammengetragenen Dinge. Im Unterschied zu klassischen Museumsobjekten wurden seine Bestände aber nicht gesammelt, um sie zu beforschen oder um an ihrem Beispiel die Geschichte der Waffentechnik und Militärgeschichte zu studieren. Das Kriegsgerät wurde erworben und eingelagert, um im Kampf genutzt zu werden, um sich damit selbst 


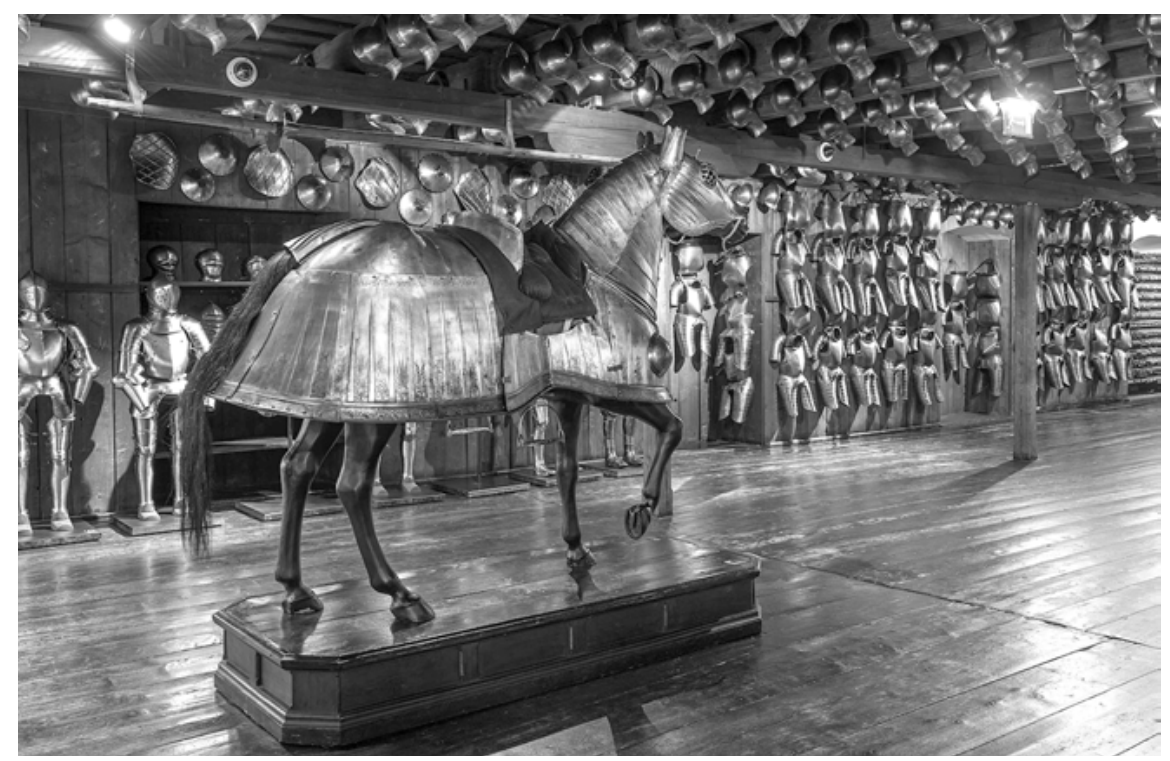

Abb. 5: Landeszeughaus.

zu schützen und um es gegen andere zu richten. Erst mit der Eingliederung des Zeughauses in das Joanneum im ausgehenden 19. Jahrhundert erhielt der Ort eine museale Rahmung: mit Eintrittskarten, Kassen- und Aufsichtspersonal, Berührungs- und Fotoverboten, Vorgaben bezüglich Wegführung und klar definierten Öffnungszeiten, die fortan die Verhaltensweisen des Publikums und die Wahrnehmung des Ortes als Museum bestimmten.

Im Gegensatz zu den Beständen des Zeughauses ist die Kulturhistorische Sammlung immer schon Herzstück und Basis eines klassischen Museums gewesen. Im Laufe des 20. Jahrhunderts in wechselnder Form präsentiert, war es dem Publikum mit dem sukzessiven Abbau der Dauerausstellung ab den 1990erJahren aber nicht mehr möglich, einen Eindruck davon zu gewinnen, was diese Sammlung beinhaltet und ausmacht, mit Folgen für die institutionelle Identität des ursprünglichen „Culturhistorischen und Kunstgewerbemuseums“. Der Umzug der Sammlung wurde schließlich zum Startpunkt einer Neuprofilierung des Hauses unter neuem Namen. Mit der Reorganisation der Dauerausstellung in den Jahren 2016 und 2017 gelangte die Sammlung in Form eines Schaudepots zurück in die Ausstellungsräume.

Schaudepots zielen grundsätzlich darauf ab, einen möglichst repräsentativen und umfassenden Einblick in Sammlungen zu gewähren (vgl. Natter et al. 2010). Hinter den zahlreichen Gründungen der letzten Jahre im gesamten deutschsprachigen Raum steht nicht nur die Attraktivität eines vermeintlichen 


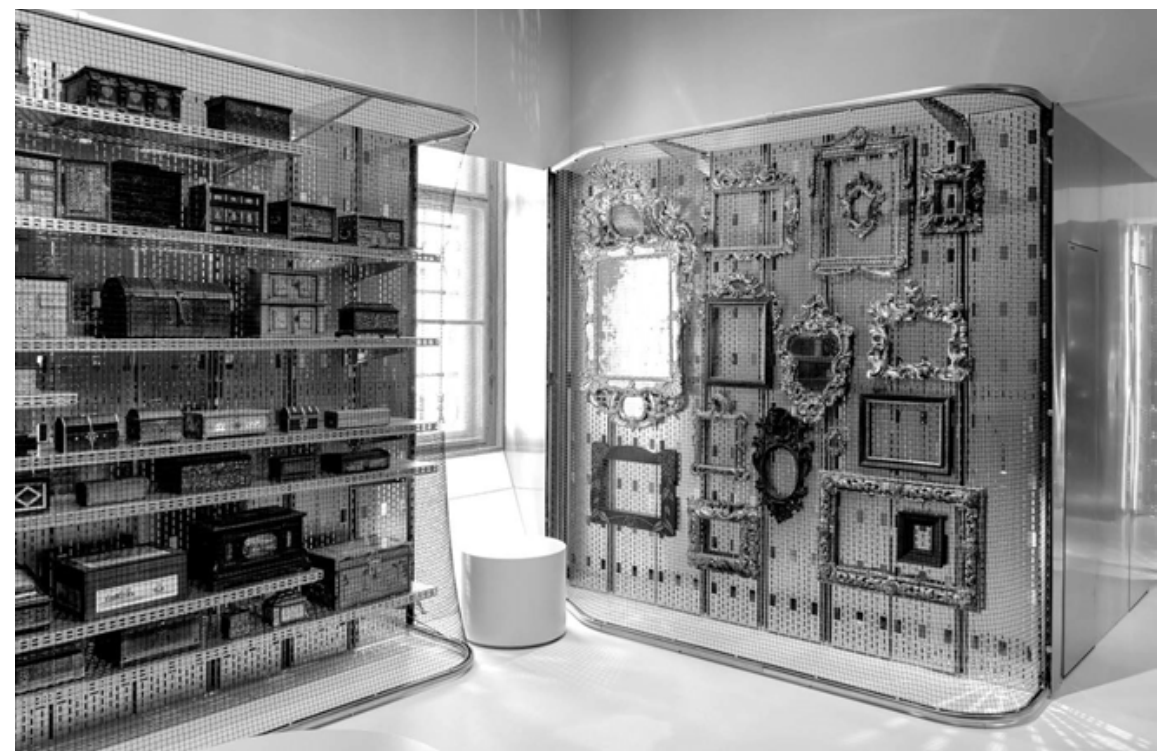

Abb. 6: Ansicht Schaudepot, Museum für Geschichte.

Depoteinblicks für das Publikum. Museumsverantwortliche möchten in Zeiten einer stark nach außen orientierten Institution mit dem organisierten Blick auf die Masse der Dinge auch dafür sensibilisieren, dass „Museum“ mehr als „Ausstellungshalle“ bedeutet und die Erhaltung der eigentlich im Depot verwahrten Bestände zu den zentralen Aufgaben der Institution Museum zählt. Die Mode der Schaudepots speist sich darüber hinaus aus einem Wandel der Dauerausstellungen im kulturhistorischen Bereich, die heute vielfach im Stil der Sonderausstellungen einfach rezipierbaren Erzählungen folgen, während Schaudepots nah an dem sind, was Schausammlungen früher waren: mehrdimensional.

Die Ordnung der Dinge im Schaudepot kann in ganz unterschiedlicher Weise, etwa nach Sammlungsbereichen, Materialien und Farben oder entlang der Buchstaben des Alphabets erfolgen. Im Sinne der BesucherInnenorientierung findet sich die Kulturhistorische Sammlung des Joanneums nach funktionalen Gesichtspunkten gegliedert, wobei die insgesamt neun Themenfelder jeweils ein bis zwei Sammlungen schwerpunktmäßig vorstellen. So werden etwa im Abschnitt „Essen \& Trinken“ exemplarisch die Bestände aus Glas, Keramik und Porzellan präsentiert. Unter „Reisen \& Fortbewegen“ findet sich ein Teil der Sammlung historischer Fahrzeuge wieder. Der Abschnitt „Arbeiten \& Produzieren“ zeigt Auszüge aus dem Fundus zum Handwerk oder jener zu „Forschen \& Wissen“ die neuzeitlichen Globen und Messinstrumente. 
Während das Schaudepot der Kulturhistorischen Sammlung das Innerste des Museums nach außen kehrt, sind das Depot und die Bestände der Multimedialen Sammlungen für die Öffentlichkeit im doppelten Sinn unzugänglich. Zum einen hat die große Zahl an prinzipiell leicht verfügbaren, massenhaft hergestellten und beliebig vervielfältigbaren Fotografien bereits seit den 1960er-Jahren zur Anhäufung von enormen Objektmengen geführt. Nachdem mit einem neuerlichen Umzug die zeitgemäße Verwahrung und Sicherung der Fotos, Film- und Tondokumente begonnen wurde, wird die inhaltliche Erfassung, Beschreibung und damit Zugänglichmachung dieser selbst bei konsequenter Arbeit weitere Jahre in Anspruch nehmen, während sich vor dem Hintergrund wechselnder Rahmenbedingungen - Stichworte wären etwa Digitalisierung oder Bildrechte laufend neue Fragen ergeben. Die Unzugänglichkeit von Depot und Sammlung ergibt sich zum anderen aus den hohen konservatorischen Ansprüchen von Foto, Film und Ton, was sowohl die Lagerung als auch die Präsentation betrifft. Obwohl die Bestände mittlerweile bei 4 und $17{ }^{\circ} \mathrm{C}$ im Klimadepot untergebracht sind, kämpfen die Verantwortlichen mit der geringen Halbwertszeit mancher Objekttypen, mit materialbedingten Unvereinbarkeiten einzelner Objektgruppen oder ihrer leichten Entflammbarkeit. Was die historischen Tonaufnahmen und Filme angeht, ist die Zugänglichkeit selbst für das Team der Sammlung an die Verfügbarkeit entsprechender technischer Ausstattung und die Anwesenheit kompetenter MitarbeiterInnen gebunden.

An der skizzierten Unzugänglichkeit zeigt sich, wie sehr sich die drei genannten Depots neben ihrer Geschichte und historischen Funktion in ihrem Verhältnis zur Öffentlichkeit unterscheiden. Die Kunst- und Wunderkammern der Frühen Neuzeit waren private Orte, der Zutritt für Außenstehende konnte nur auf eine Einladung hin erfolgen. Gleich ob nun politischer Repräsentant, Gelehrter oder Bildungsreisender - die Gäste wurden vom Sammler persönlich oder einem fachkompetenten Vertreter begrüßt und durch die Räumlichkeiten geführt. Die Befassung mit den ausgestellten Dingen erfolgte in Form von Gesprächen und auf Augenhöhe, unter Wissenden und Experten, die im Austausch die Sammlungen qualitativ weiterentwickelten.

Dagegen stand das Museum der Moderne grundsätzlich allen offen. Dies stimmte bedingt: Zwar vergrößerte sich die Zahl jener, die Museen besuchten, weite Teile der Bevölkerung blieben aber aufgrund von Eintrittsgeldern, beschränkten Öffnungszeiten und Bekleidungsvorschriften außen vor. Mit Erfindung des Museums änderte sich auch die Praxis des Besuchs: Wie Sharon McDonald ausführt, war im modernen Museum nicht das Gespräch, sondern das Sehen zentral, um Wissen über die Objekte zu erlangen (vgl. McDonald 2003, 3-4). Die gläsernen Schaumöbel gaben ihr Inneres ohne Einschränkungen preis und machten sichtbar. Sie hielten die BetrachterInnen aber gleichzeitig auf Distanz, 

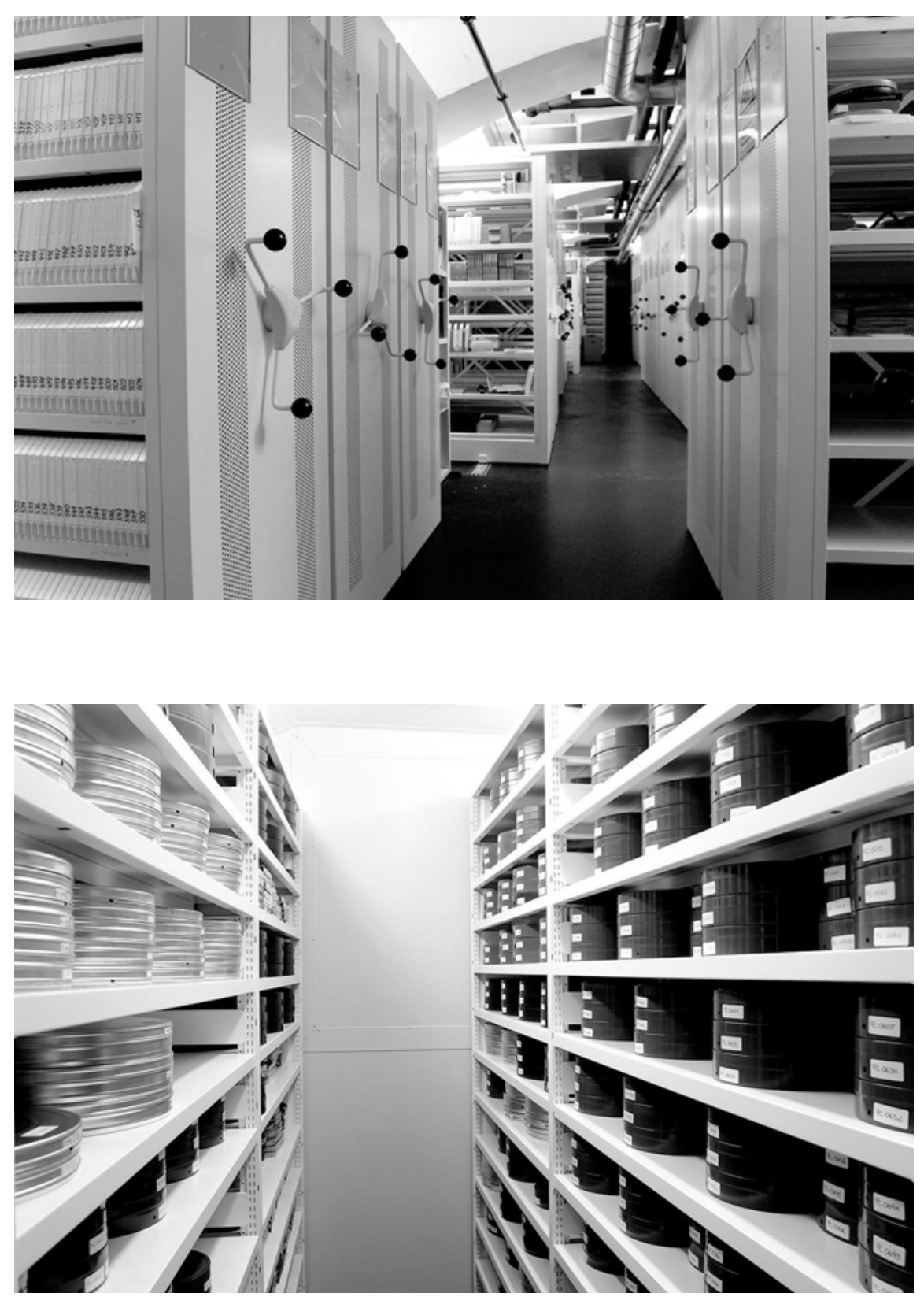

Abb. 7-8: Depot Multimediale Sammlungen. 
wenn diese von außen oder oben auf eine geordnete Welt blickten. Die klare Gliederung der Objekte objektivierte - so McDonald - die wissenschaftlichen Erkenntnisse für das Publikum, machte sie glaubwürdig, zwingend wahr, nicht hinterfragbar. Wissenschaftler und Publikum begegneten sich im Museum nicht mehr auf Augenhöhe, sondern in einer hierarchischen Situation.

Das Landeszeughaus war nie für die Öffentlichkeit bestimmt, sondern als geheimer Ort lediglich für einen kleinen Kreis ausgewählter Personen einsehbar. Öffentlichkeit, eben das, was Museum seit seinen Anfängen ausmacht, war dort, um Spionage zu verhindern, strikt untersagt! Die Multimedialen Sammlungen unterliegen keiner Geheimhaltung, hier wird die Öffentlichkeit aber aus Gründen des Objektschutzes ausgesperrt - die winterlichen Temperaturen in den Klimadepots leisten einen Beitrag zur Akzeptanz der Regelung. Auch ist eine dauerhafte Zurschaustellung der Bestände undenkbar: Die Möglichkeit einer Präsentation historischer Fotografien ist auf kurze Zeitfenster beschränkt, die konservatorischen Auflagen machen es kompliziert. Diese radikale Beschränkung der Zugänglichkeit betrifft auch Forschende: Ein Stöbern und Suchen gestaltet sich angesichts der Objektmengen und der Temperaturen in den Klimadepots als schwierig. Eine Komplett-Digitalisierung ist angesichts der Kosten und der entstehenden Datenberge unrealistisch, eine projektorientierte, punktuelle Erschließung, Digitalisierung und Veröffentlichung laufendes Programm. Im Schaudepot der Kulturhistorischen Sammlung schließlich steht die Entscheidung für eine größtmögliche Öffentlichkeit und Zugänglichkeit in Spannung zu den konservatorischen Vorgaben und den Ansprüchen der Objektsicherung. Zugänglichkeit bedeutet im Fall des Schaudepots aber mehr als die Befreiung der Objekte aus den Engen des Depots. Sie zeigt sich im Verzicht auf Vitrinen und Glaswände sowie der Entscheidung für eine möglichst zeitgemäße Gestaltung, die Schleifkannen und Lichtputzscheren in einer Weise rahmt, dass Menschen von heute mit ihren spezifischen Wahrnehmungsgewohnheiten Lust haben, ihnen Aufmerksamkeit zu schenken.

Wie reagiert nun das Publikum konkret? Wie steht es um die Praxis des Besuchs, vergleicht man das Landeszeughaus mit dem Schaudepot im Museum für Geschichte? Wie die Erfahrung zeigt, wird ganz generell die offene Präsentation möglichst vieler Objekte von den Besucherinnen und Besuchern als attraktiv und ansprechend empfunden. Dahinter steht der Reiz, vermeintlich Zugang zu in der Regel unzugänglichen Dingen zu erhalten, ein gewisser „Dachbodeneffekt“, der auf Überraschungen hoffen lässt, die Idee von Exklusivität ob der Möglichkeit, aus einer Fülle nach Belieben selbst auswählen zu können, die Attraktivität des Seriellen oder an einem Ort konzentriert zu sehen, wie sich Geschichte seit Jahrhunderten materialisiert, schließlich die Vorstellung, Objekte in einem nur scheinbar vor-kuratorischen Moment anzuschauen. Schaudepots entsprechen auch einer aktuellen Praxis des Konsums, dem Shoppen und Zappen, einer 
Lebenshaltung, nach der man auf alles zugreifen, über alles verfügen, alles potenziell mit allem zusammenbringen kann.

Eine Beschriftung der Objekte gibt es im Schaudepot der Kulturhistorischen Sammlung aufgrund der hohen Objektzahl nicht. Eine inhaltliche Orientierung und Beschreibung erfolgt in einem knappen Gesamtverzeichnis aller Objekte, in einer kostenfrei zugänglichen Begleitbroschüre sowie in Form von Überblicksund Themenführungen. Die Annahme, dass sich Menschen ohne Ablenkungen durch Texte, begleitende Filme etc. intensiv den Objekten widmen würden, trifft nur für einen Teil des Publikums zu. Auf der anderen Seite stehen die Neugierigen, Wissbegierigen und schließlich Verunsicherten, die sich von Datierung und Herkunftsangaben als bekannte museale Kategorien eine Hilfestellung erhoffen, um sich von einem Gegenstand überhaupt einen Begriff machen zu können. Bemerkenswert ist zudem, dass - nachdem die letzte Dauerausstellung lediglich rund 200 Objekte umfasste - nun die Masse der Dinge den Wunsch nach noch mehr Dingen provoziert und immer wieder der Verbleib einmal gesehener Sammlungsstücke nachgefragt wird.

Im Zeughaus ist das Publikumsverhalten ein anderes. Viele kommen ohne konkrete Erwartungshaltung an den Ort, der mehr oder weniger fixer Bestandteil eines touristischen Graz-Besuchs ist, und sind dann überwältigt. Einmal eingetreten, geraten die Gäste innerhalb kurzer Zeit in ein Staunen und Taumeln, aus dem sie sich dann über vier Etagen nur schwer befreien können. Warum ist das so? Das Zeughaus ist ein immersiver, stark vereinnahmender Stimmungs- und Atmosphärenraum. Was in kommerziellen Erlebnis- und Einkaufswelten planvoll inszeniert wird, findet sich im Zeughaus unbeabsichtigt umgesetzt: die Größe der Anlage, die Geschlossenheit der Räume, die mit dem Tages- und Jahreslauf korrespondierende Lichtsituation und Temperatur, die Materialität und Farbigkeit der massenhaft in Reih und Glied bereitgestellten Waffen, der Geruch des Holzes, das Knarren der Böden - all diese Elemente tragen dazu bei, dass das Publikum in den Raum eintaucht und sich im Gang durch die Etagen verliert.

Dies wäre nun weniger problematisch, würde das Zeughaus nicht eine heikle Sammlung beherbergen. In Museumskontexten hat sich in den letzten Jahren der Begriff der „sensiblen Sammlung“ (Berner et al. 2011) zur Beschreibung menschlicher Überreste in archäologischen oder medizinhistorischen Sammlungen (wie Knochen, Schädel etc.) sowie Objekten aus sogenannten Unrechtskontexten (Raubgut aus Kolonialgeschichte, Kriegshandlungen u. ä.) durchgesetzt. Obwohl nun die Bestände des Landezeughauses dieser Definition im engeren Sinn nicht entsprechen, scheint der Begriff doch insofern brauchbar, als es sich bei den versammelten Objekten zu einem beachtlichen Teil um Tötungsinstrumente handelt, deren eigentliche Bestimmung hinter ihrer ästhetischen Wirkung allzu leicht verblasst. Dies bewusst $\mathrm{zu}$ machen ist wesentliche Aufgabe des BesucherInnen- 
service. Auch weil in die architektonischen Gegebenheiten und die Aufstellung der Sammlung aus Gründen des Denkmalschutzes nicht eingegriffen werden kann, liegt es an den Vermittlerinnen und Vermittlern, den Blick auf die Bestände zu organisieren, bestimmte Perspektiven, neues Wissen, Haltung einzubringen. Begleitbroschüren, Medienstationen und Audioguides unterstützen dabei, den Blick auf das Dargebotene zu diversifizieren und seinen militärhistorischen, sozialgeschichtlichen, restauratorischen oder museologischen Gehalt aufzuschließen.

\section{Literaturverzeichnis}

Berner, Margit, Anette Hoffmann und Britta Lange. Sensible Sammlungen. Aus dem anthropologischen Depot. Hamburg: Philo Fine Arts, 2011.

Groys, Boris. „Archiv der Zukunft. Das Museum nach seinem Tod“. Die Aneignung der Vergangenheit. Musealisierung und Geschichte. Hg. Ulrich Borsdorf, Heinrich Theodor Grütter und Jörn Rüsen. Bielefeld: transcript, 2005. 39-79.

Heesen, Anke te. „Geschlossene und transparente Ordnungen. Sammlungsmöbel und ihre Wahrnehmung in der Aufklärungszeit“. Wahrnehmung der Natur. Natur der Wahrnehmung. Studien zur Geschichte visueller Kultur um 1800. Hg. Gabriele Dürbeck, Bettina Gockel, Susanne B. Keller, Monika Renneberg, Jutta Schickore und Gerhard Wiesenfeldt. Amsterdam, Dresden: Verlag der Kunst, 2001.19-34.

McDonald, Sharon. „Museums, national, postnational and transcultural identities“. Museum and Society 1.1 (2003): 1-16.

Möbius, Hanno. „Konturen des Museums im 19. Jahrhundert (1789-1918)“. Zur Geschichte der deutschen Museen im 19. Jahrhundert 1789-1918. Hg. Bernhard Graf und Hanno Möbius. Berlin: G-und-H-Verlag, 2006. 11-22.

Natter, Tobias, Michael Fehr und Bettina Habsburg-Lothringen (Hg.). Das Schaudepot. Zwischen offenem Magazin und Inszenierung. Bielefeld: transcript, 2010. 
